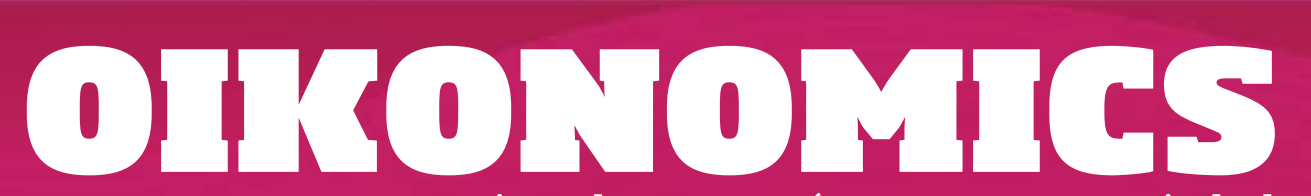

Revista de economía, empresa y sociedad

CAMBIO EN LA CULTURA EMPRESARIAL

\title{
La fintegración del sistema bancario
}

\section{Āngels Fitó Bertran}

Directora de los Estudios de Economía y Empresa (UOC)

RESUMEN Las bases sobre las que se ha asentado el negocio financiero tradicional están siendo sacudidas por la aparición de nuevos actores que introducen nuevos modelos de negocio sustentados en las oportunidades que ofrecen los recientes avances tecnológicos. Las fintech son empresas de base tecnológica que ofrecen servicios financieros digitalmente, mediante soluciones tecnológicas y con un enfoque centrado en las necesidades y preferencias del consumidor. Ante la amenaza que supone esta disrupción en el mercado financiero, bancos y fintech están desarrollando estrategias de colaboración que aprovechan el potencial innovador de las fintech para llevarlo al gran público a través de las estructuras y carteras de la banca tradicional. Este proceso de integración no está exento de obstáculos y retos, entre los cuales destaca el cambio en la cultura empresarial de las entidades financieras tradicionales.

PALABRAS CLAVE fintech; sector financiero; banca; innovación digital

\section{The fintegration of the banking system}

ABSTRACT The foundations of traditional financial business are being shaken by the emergence of new actors who are introducing new business models based on the opportunities offered by recent technological advances. Fintechs are technology-based companies that offer financial services digitally through technological solutions and which focus on the needs and preferences of the consumer. Faced with the threat posed by this disruption 
in the financial market, banks and fintechs are developing collaboration strategies that take advantage of the innovative potential of fintechs in order to bring it to the general public through traditional banking structures and portfolios. This integration process is not free from obstacles and challenges, one of the main ones being the change in the business culture of traditional financial institutions.

KEYWORDS fintech; financial sector; banking; digital innovation

\section{Introducción}

El desarrollo de la tecnología ha transformado el sector financiero, de modo que han proliferado nuevas iniciativas que, mediante soluciones digitales innovadoras, ofrecen servicios financieros a millones de usuarios. Nos referimos al ecosistema fintech. A continuación, a partir del estudio de distintos informes y trabajos publicados, se analiza la trayectoria de estas empresas y su proceso de integración con el sistema bancario tradicional. Al final del recorrido, se pone de manifiesto cómo la colaboración entre la banca y las fintech se percibe como la mejor estrategia para seguir compitiendo en un mercado donde la tecnología seguirá planteando nuevos retos.

\section{La transformación digital del sector financiero}

El hecho de que la tecnología ha propiciado una transformación de gran calado en el sector financiero tradicional es una obviedad. Con el fin de aprovechar las oportunidades que la innovación tecnológica ofrece en términos de acceso, coste y seguridad, tanto la oferta como la demanda de servicios financieros han adaptado sus procesos y hábitos de consumo, si bien no siempre a la misma velocidad. Tal y como destaca el informe del Observatorio de Digitalización Financiera (ODF) sobre la toma de decisiones financieras de los españoles en un contexto de transformación digital (1), las entidades financieras españolas, probablemente empujadas por los nuevos modelos de negocio que emergen bajo sus pies, han llevado a cabo un enorme esfuerzo para transformarse digitalmente. A su vez, en la demanda de servicios financieros también se detectan cambios en los patrones de consumo que permiten hablar de un nuevo cliente digital, con unos rasgos y comportamientos diferenciados y que exige a sus proveedores de servicios mejor acceso, mayor personalización y más transparencia. 
En lo que llevamos de milenio, este consumidor de servicios financieros ha evolucionado de manera significativa y ha pasado de lo que se podría considerar un cliente sucursalizado, uno fuertemente arraigado al pago en efectivo y con una percepción de la banca en línea como un servicio disponible, ágil, pero poco seguro, al consumidor actual, que está permanentemente conectado, que hace uso de la banca en línea para consultar saldos o movimientos y hacer transferencias, y que transita de la banca en línea a la banca móvil por ser un entorno más cómodo y seguro. En este camino hacia la digitalización de los servicios financieros, todavía con un largo recorrido por delante, las proyecciones del ODF apuntan hacia una ampliación del abanico de actividades que se harán en línea, la imposición definitiva del móvil para llevar a cabo dichas actividades, así como la incursión de nuevos intermediarios no bancarios que deberán abastecer a un consumidor cada vez más empoderado.

\section{El ecosistema fintech}

Sin embargo, el impacto más destacado que esta revolución tecnológica ha ocasionado en el sector financiero no se encuentra tanto en el cambio de métodos y comportamientos de bancos y clientes, sino precisamente en la irrupción de nuevos actores que, blandiendo estrategias de negocio disruptivas, se están haciendo un lugar en el sofisticado mercado de las finanzas. Estamos hablando del ecosistema fintech, donde proliferan un conjunto de iniciativas, a menudo concebidas como startups, que hacen un uso extensivo y disruptivo de la tecnología para ofrecer distintos servicios financieros que evitan la intermediación bancaria.

En este escenario, el informe «Fintech, Innovación al servicio del cliente» (2), elaborado conjuntamente por Funcas y KPMG, describe las empresas que cohabitan en este entorno como entidades que (a) ofrecen productos financieros totalmente en línea a través de (b) tecnologías disruptivas, estructuras flexibles y metodologías ágiles y (c) con un enfoque centrado en las necesidades y preferencias del consumidor. (d) Se basan en un modelo desintermediado y esto (e) favorece la inclusión financiera de segmentos de la población al democratizar el acceso a más servicios financieros, a la vez que (f) reduce el coste de la prestación del servicio a través de sistemas más eficientes.

El catálogo de servicios que ofrecen estas organizaciones a un consumidor cada vez más conectado, informado, y por lo tanto más exigente, no deja de crecer. Hoy día, existen múltiples e imaginativas fórmulas financieras que dan acceso a financiación a millones de usuarios y empresas mediante soluciones digitales. Mientras los tipos de interés del BCE están por los suelos y los bancos siguen imponiendo tipos próximos a las dos cifras en los préstamos al consumo, el crowdlending, 
o préstamos colectivos, ocupa la primera posición en el ranking de las finanzas colaborativas. A su vez, en un contexto donde las empresas han asistido impotentes al cierre del grifo del crédito, el crowdfunding, o inversiones o préstamos colectivos vinculados al éxito del proyecto, está desbancando el negocio del capital-riesgo clásico. Sin embargo, estas son solo dos, las más grandes, de las fórmulas que se han desarrollado en el ámbito de las finanzas colaborativas. Depósitos en cuentas PayPal, criptomonedas como el bitcoin, servicios financieros como los que ya ofrecen gigantes tecnológicos como Facebook, Amazon, Google, Apple o Alibaba; los Neobanks que utilizan exclusivamente las redes sociales para relacionarse con sus clientes, o incluso plataformas aseguradoras donde la buena conducción del colectivo asegurado revierte en las cuotas del grupo, son algunas de las nuevas iniciativas y actores que día a día aumentan exponencialmente el número de adeptos.

\section{Los financieros tradicionales frente a los financieros disruptivos}

Las últimas cifras ofrecidas por la Universidad de Cambridge (3) en su segundo informe europeo sobre la situación y evolución de la industria financiera alternativa ponen de manifiesto que en Europa ha existido un crecimiento más que exponencial de este ecosistema alternativo de soluciones financieras. El documento, que lleva por título "Sustaining Momentum», destaca que durante el periodo 20132015, el volumen de transacciones en Europa ha superado los 5.000 millones de $€$. El informe apunta a que el peso de esta industria es aún pequeño, y que la ralentización de su crecimiento en el último año es el reflejo de los riesgos que esta industria debe enfrentar para transitar de la startup a formas sostenibles de financiación dentro del ecosistema de servicios financieros europeos. En el ámbito nacional, las últimas cifras del ODF del 2017 (2) sitúan a España, con aproximadamente 300 empresas, en el sexto país del mundo por número de fintechs. Este sector emergente ocupa a más de 3.500 trabajadores, y la facturación asciende a más de 100 millones de euros. Con la quinta posición mundial en cuanto al uso de productos y servicios fintech por parte de los consumidores, se estima que la nueva inversión en el 2017 fue de 250 millones. Esta inversión se ve atraída por las características del mercado español, fuertemente bancarizado, con un sector bancario que en los últimos años ha sufrido un destacado proceso de concentración y en el que el móvil está alcanzando elevados índices de penetración.

¿Y qué piensan los financieros tradicionales de los financieros disruptivos? Un informe de The Economist, «The disruption of banking» (4), resumió las opiniones de más de 100 ejecutivos sénior de entidades financieras y la misma cifra de ejecutivos de empresas fintech. Resulta revelador destacar que más del $90 \%$ de los 
banqueros entrevistados reconocieran que las fintech tendrían un impacto significativo sobre el sector. El efecto de la fintegración, que empezó por los propios servicios de banca en línea, fue solo el inicio de los cambios en el comportamiento de los consumidores financieros. Sin embargo, resulta sorprendente que el $54 \%$ de los entrevistados afirmaran que, si bien ya hace tiempo que las entidades financieras hablan de disrupción, no están aún implantando cambios significativos en esta línea. En cuanto a las debilidades que más claramente pueden dañar el posicionamiento de la banca tradicional, destacan «una visión digital, clara y estratégica», «el peligro de violaciones de seguridad» y «una cultura que se adapte rápidamente al cambio». La otra cara de la moneda, las fortalezas que aún atesoran los bancos, es «la reputación de estabilidad», «la lealtad del cliente» $y$ «la experiencia regulatoria y en la gestión de riesgos».

¿Y cuál es la perspectiva de las fintech? Casi la mitad de sus ejecutivos reconoce que los bancos seguirán dominando el sector, si bien más de la cuarta parte sugiere que será un mix equilibrado de las dos fórmulas lo que imperará en el mercado. En cuanto a las debilidades, las fintech reconocen la inexperiencia en la gestión de riesgos, un nivel insuficiente de capital invertido, la falta de experiencia directiva y la escasa confianza del cliente ante soluciones tan novedosas como aparentemente poco seguras. Sin embargo, la especialización en determinados productos, la ausencia de regulación en determinados segmentos, la agilidad para llevar al mercado nuevas propuestas y la capacidad de innovación son algunas de las bazas que juegan a su favor.

Es evidente que la estructura ágil e innovadora de las startups financieras les ha permitido hacerse un hueco en el ecosistema financiero, haciendo llegar al mercado propuestas creativas que se ajustan mejor a las expectativas de los consumidores finales. Su capacidad de dar respuesta a necesidades que las grandes entidades bancarias tradicionales no han sido capaces de abordar les ha otorgado el papel de motor de la innovación en el sector financiero. Por su parte, la banca tradicional, que cuenta en su haber con la experiencia y también con la confianza, tiene además lo más importante para alcanzar grandes cuotas de mercado: los clientes (las últimas cifras publicadas en el «Barómetro de Innovación Financiera» (5) muestran que, en el mercado español, existe una importante vinculación del cliente con su entidad financiera principal, siendo 1,7 el número medio de entidades con las que un cliente tiene relación).

\section{La fintegración del sector financiero}

A finales del milenio pasado, Bill Gates vaticinó que en un futuro los servicios financieros serían necesarios, pero no los bancos. Si bien resulta evidente que los ban- 
cos deben abordar un profundo proceso de transformación, pero no solo en temas tecnológicos, puesto que la tecnología por sí sola no es intrínsecamente disruptiva, también es cierto que las fintech necesitan aún ganarse la confianza de un público mucho más numeroso para poder garantizar su continuidad. Frente a la hipotética lucha por imponerse en el mercado, se podrían plantear diferentes escenarios que pasan por una cooperación en la que los bancos aprovechen los avances de las fintech para mejorar la experiencia de sus consumidores, una posible fragmentación o reparto de los servicios financieros, o bien una residualización del papel de los bancos, que perderían su función de intermediación bancaria, dejando el trato con el cliente en manos de las fintech. Sin embargo, parece que en la batalla entre el sector financiero tradicional y las fintech, finalmente se va acabar imponiendo el dicho «si no puedes con el enemigo, únete a él».

Así, es precisamente en un contexto tan altamente competitivo y complejo como el mercado financiero donde parece que se impone, por el momento, una estrategia de cooperación. Los últimos informes publicados ponen de relieve que las nuevas propuestas revolucionarias de las fintech se vuelven rentables cuando cuentan con la estructura y la cartera de los grandes bancos para implantarlas de forma masiva. Esta conclusión se desprende de un estudio publicado también por el ODF (6) en el que, entre otras afirmaciones, se apunta que el $75 \%$ de los bancos llevan a cabo acuerdos con empresas fintech, el 56 \% están desintermediando su modelo de negocio y el 75 \% efectúan acuerdos de cocreación. A su vez, desde el punto de vista de las fintech, un $26 \%$ de las mismas manifiestan haber alcanzado su punto muerto gracias, en parte, a las alianzas selladas con la banca tradicional.

Este proceso de integración, que ya ha dado sus primeros pasos, no está exento de obstáculos. El Global Fintech Report (7), publicado por PwC en el 2017, alerta de un conjunto de elementos que deben ser tenidos en consideración para afrontar procesos de colaboración o participación entre instituciones financieras y empresas de fintech. Las diferencias culturales y en los modelos de gestión, la incertidumbre ante un marco regulatorio por establecer y los contrastes entre los distintos modelos de gestión son las principales dificultades que cualquier alianza entre socios financieros deberá afrontar. A continuación, le siguen aspectos de carácter más técnico como las incompatibilidades tecnológicas, las discrepancias en los procesos operativos, las diferencias en competencias tecnológicas o bien aspectos relativos a la seguridad tecnológica.

La irrupción constante de nuevas tecnologías como la blockchain o la inteligencia artificial, entre otras, es precisamente lo que espolea la implantación de estrategias activas de colaboración banca-fintech y permite a los dos estar a la vanguardia de la innovación tecnológica. Por lo tanto, la historia de convivencia de estos dos modelos de negocio, tradicional y disruptivo, ha trascurrido desde una etapa inicial en la que se preveía una feroz lucha para hacerse o mantener cuotas de mercado, 
al estadio actual, en el que alianzas y acuerdos de colaboración parecen mantenerlas a salvo de un enemigo claramente superior, las big tech, o los gigantes tecnológicos popularmente conocidos como GAFA (Google, Apple, Facebook y Amazon) o sus colegas asiáticos BAT (Baidu, Alipay y Tencent).

\section{Los retos de la cooperación financiera}

Cuando las nuevas generaciones se conviertan en consumidores de productos financieros, exigirán a sus proveedores los mismos niveles de servicio e innovación que encuentran en Google o Amazon. Ante este escenario, para continuar siendo competitivos y no ser devorados por un mercado que puede verse arrastrado por las tendencias oligopólicas que imponen los procesos de digitalización, las alianzas financieras deberán poner énfasis en aquellas políticas que promuevan la innovación. En este sentido, el Global Fintech Report (7), a partir de los resultados de una encuesta a 1.308 directivos de entidades financieras y fintech, identifica un conjunto de seis factores que deben incluirse en las estrategias de innovación.

En primer lugar, la exploración de estrategias de asociación en las cuales la complementariedad de competencias y talentos puede derivar en importantes sinergias de negocio. En segundo lugar, la continua evaluación de las tecnologías emergentes que estimula la alianza banca-fintech, ya sea incubando o acelerando nuevas startups tecnológicas que testeen nuevos sistemas de aproximación a los clientes, se concibe como una estrategia de futuro. En tercer lugar, la integración de las innovaciones tecnológicas que actualicen los sistemas existentes y los doten de mayor agilidad. A continuación, el establecimiento de una cultura tecnológica, pero también organizativa, que estimule y dé apoyo a la innovación. También se precisa de una mayor focalización al comportamiento, necesidades y preferencias del consumidor, dándole voz en los procesos de innovación. Finalmente, resulta imprescindible promover una cultura empresarial que dé apoyo al talento y la innovación.

\section{Conclusiones}

Las principales conclusiones que se extraen de este sucinto recorrido por la situación del mercado de los servicios financieros son las que se exponen a continuación:

- El sector financiero está viviendo un intenso proceso de transformación digital, en el que las fintech ejercen el papel de disruptor tecnológico impulsando el proceso de transformación tecnológica del sector bancario tradicional. 
- Tras un periodo inicial de desconcierto y recelo por parte del sector tradicional, se imponen estrategias de colaboración en las cuales la innovación que se gesta en las fintech se industrializa a través de las estructuras de la banca tradicional.

- La principal amenaza a la que se enfrentan actualmente banca y fintech son los denominados bigtech, que atraen a grandes inversores y atesoran la confianza de millones de usuarios. En un futuro, las previsiones son también de una mayor colaboración entre las fintech y los gigantes tecnológicos.

- Las estrategias de colaboración o integración entre la banca tradicional y las fintech deben promover la innovación tecnológica y la compartición de conocimiento, así como fomentar una nueva cultura organizativa que dé alas a la innovación tecnológica y empresarial.

\section{Referencias bibliográficas}

(1) OBSERVATORIO DE DIGITALIZACIÓN FINANCIERA FUNCAS-KPMG (2017). «¿Cómo toman los españoles sus decisiones financieras digitales?» [en línea]. <https://home.kpmg.com/es/es/home/sala-de-prensa/notas-de-prensa/2017/11/como-toman-espanoles-decisiones-financieras-digitales.html>

(2) OBSERVATORIO DE DIGITALIZACIÓN FINANCIERA FUNCAS-KPMG (2017). «Fintech, innovación al servicio del cliente». <https://assets.kpmg.com/content/dam/kpmg/es/pdf/2017/11/fintech-innovacion-servicio-cliente.pdf>

(3) CAMBRIDGE CENTRE FOR ALTERNATIVE FINANCE (2016). «Sustaining Momentum, The 2nd European Alternative Finance Industry Report». [en línea]. <https://www.jbs.cam.ac.uk/fileadmin/user_upload/research/centres/alternative-finance/downloads/2016-european-alternative-finance-report-sustaining-momentum.pdf>

(4) THE ECONOMIST INTELLIGENCE UNIT LIMITED (2015). «The disruption of banking» [en línea]. <https://www.eiuperspectives.economist.com/sites/default/files/EIU-The\%20disruption\%20of\%20banking_PDF_0.pdf>

(5) FUNCAS; FINNOVATING (2018). «Barómetro de Innovación Financiera» [en línea]. <http://www.finnovating.com/report/i-barometro-de-innovacion-financiera-2018/>

(6) OBSERVATORIO DE DIGITALIZACIÓN FINANCIERA FUNCAS-KPMG (2017). «El nivel de madurez digital del sector financiero español». Observatorio de Digitalización Financiera.

(7) PWC (2017). «Redrawing the lines: Fintech's growing influence of Financial Services». Global Fintech Report 2017 [en línea]. <https://www.pwc.com/gx/en/ industries/financial-services/assets/pwc-global-fintech-report-2017.pdf> 


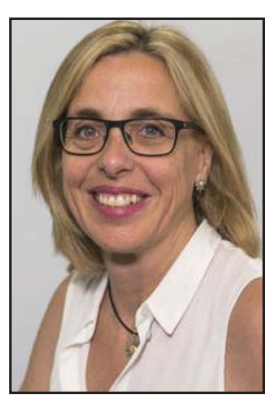

\section{Àngels Fitó Bertran afitob@uoc.edu Directora de los estudios de Economía y Empresa (UOC)}

Doctora en Contabilidad y Auditoría por la Universidad de Barcelona y máster en Dirección fiscal y financiera de la empresa (UB).

Directora de los estudios de Economía y Empresa de la UOC. Durante años, ha compaginado la docencia e investigación en contabilidad y gestión en distintas universidades con la consultoría empresarial. Sus líneas de investigación se centran en el estudio de las nuevas herramientas de gestión, el proceso de convergencia de la normativa contable internacional, así como el impacto del aprendizaje electrónico en la empresa. El fruto de esta investigación son distintas publicaciones y ponencias en congresos nacionales e internacionales.

Los textos publicados en esta revista están sujetas -salvo que se indique el contrario- a una licencia de Reconocimiento 3.0 España de Creative Commons. Podéis copiarlos, distribuirlos, comunicarlos públicamente y hacer obras derivadas siempre que reconozcáis los créditos de las obras (autoría, nombre de la revista, institución editora) de la manera especificada por los autores o por la revista. La licencia completa se puede consultar en http://creativecommons.org/licenses/by/3.0/es/deed.ca.

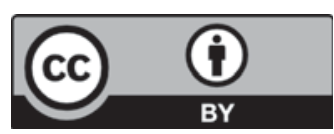

